\title{
A Phytopharmacological Review on a Medicinal Plant: Juniperus communis
}

\author{
Souravh Bais, ${ }^{1}$ Naresh Singh Gill, ${ }^{2}$ Nitan Rana, ${ }^{2}$ and Shandeep Shandil ${ }^{2}$ \\ ${ }^{1}$ Department of Pharmacology, Rayat Institute of Pharmacy, Vpo Railmajra, Nawanshahr District, Punjab 144533, India \\ ${ }^{2}$ Rayat Institute of Pharmacy, Vpo Railmajra, Nawanshahr District, Punjab 144533, India
}

Correspondence should be addressed to Souravh Bais; souravh2008.123@rediffmail.com

Received 10 June 2014; Revised 28 September 2014; Accepted 15 October 2014; Published 11 November 2014

Academic Editor: Ronaldo F. do Nascimento

Copyright (C) 2014 Souravh Bais et al. This is an open access article distributed under the Creative Commons Attribution License, which permits unrestricted use, distribution, and reproduction in any medium, provided the original work is properly cited.

\begin{abstract}
Juniperus communis is a shrub or small evergreen tree, native to Europe, South Asia, and North America, and belongs to family Cupressaceae. It has been widely used as herbal medicine from ancient time. Traditionally the plant is being potentially used as antidiarrhoeal, anti-inflammatory, astringent, and antiseptic and in the treatment of various abdominal disorders. The main chemical constituents, which were reported in J. communis L. are $\alpha$-pinene, $\beta$-pinene, apigenin, sabinene, $\beta$-sitosterol, campesterol, limonene, cupressuflavone, and many others. This review includes the last 20 years journals and various books update on this plant, representing its pharmacological activity and health benefits against various diseases.
\end{abstract}

\section{Introduction}

Plants have been used as primary sources of disease treatments from ancient times and till to date a number of species have been reported to possess various pharmacological activities [1-3]. From ancient time herbs had been used by all cultures of the world including India that has one of the oldest, richest, and most diverse culture [4]. Advances in clinical research and quality control showed a greater value of herbal medicine in the treatment and overcome from many diseases [5]. Juniperus genus is a well-known source of cedarwood oil which is widely distributed in the North hemisphere and it is used in folk medicine [6,7]. J. communis L. (Figure 1) is a shrub or small evergreen tree belonging to family Cupressaceae. The plant has been reported as diuretic, having anti-inflammatory properties $[8,9]$, antifungal activity [10], analgesic activity [11], hepatoprotective activity [12], antidiabetic and antihyperlipidemic activity [13], antimicrobial activity [14], antioxidant activity [15], antihypercholesterolemic activity [16], antibacterial activity [17], anticataleptic activity, and neuroprotective activity in Parkinson's disease $[2,18]$. The analysis of the volatile fraction of $J$. communis berries was done by HS-SPME coupled to GC/MS for gin aromatization and more than 20 constituents have been reported [19].

\section{Synonyms}

(i) Sanskrit: Havusa, Matsyagandha

(ii) Assamese: Arar, Abahal, Habbul

(iii) Bengali: Hayusha

(iv) Eng: Juniper Berry, Common Juniper

(v) Gujrati: Palash

(vi) Hindi: Havuber, Havubair

(vii) Kannada: Padma Beeja

(viii) Marathi: Hosh

(ix) Punjabi: Havulber

(x) Telugu: Hapusha

(xi) Urdu: Abhal, Aarar.

\section{Scientific Classification}

(i) Species: Juniperus communis

(ii) Class: Pinopsida

(iii) Division: Pinophyta

(iv) Order: Pinales 


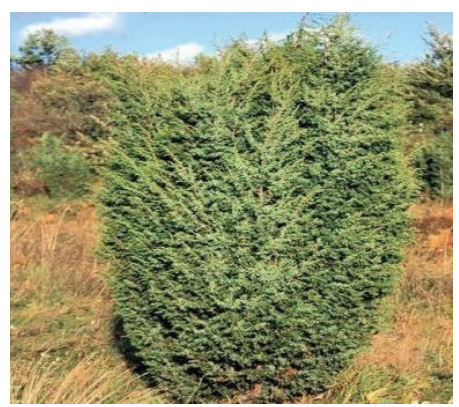

J. communis $\mathrm{L}$.

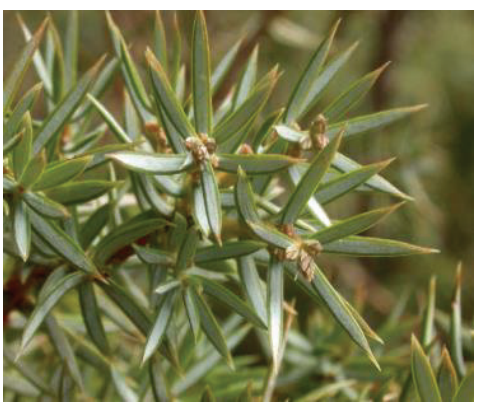

Juniperus leaves

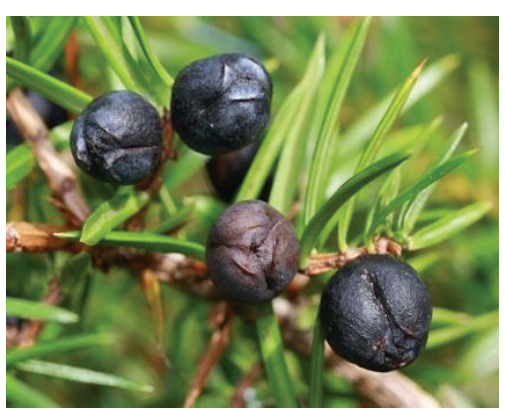

Juniperus berries

FIgURE 1: Images of J. communis L. plant.

TABLE 1: Traditional uses of J. communis L. plant.

\begin{tabular}{ll}
\hline Part & Traditional use \\
\hline Berries & $\begin{array}{l}\text { Carminative, urinary antiseptic, diuretic, emmenagogue, sudorific, digestive, and } \\
\text { anti-inflammatory. }\end{array}$ \\
Aerial parts & $\begin{array}{l}\text { Used for acute and chronic cystitis, albuminuria, catarrh of the bladder, renal suppression, } \\
\text { leucorrhoea, and amenorrhoea. }\end{array}$ \\
Fruit & $\begin{array}{l}\text { Used as antiseptic, stimulant, disinfectant, styptic, chronic Bright's disease, migraine, dropsy, } \\
\text { rheumatic and painful swellings, piles, and infantile tuberculosis. }\end{array}$ \\
Bephrotic dropsy of children, asthma, gonorrhoea, pulmonary blennorrhoea, arthritis, \\
Bark
\end{tabular}

(v) Family: Cupressaceae

(vi) Genus: Juniperus

(vii) Binomial name: J. communis L.

\section{Distribution}

J. communis is found in Himachal Pradesh at an altitude of $3000 \mathrm{~m}-4200 \mathrm{~m}$. It is mainly distributed in Manimahesh in Chamba, Kullu, Churdhar in Sirmour, Chhota and Bara Bhnghal in Kangra, and Kinnaur and Pattan valley in LahaulSpiti districts. The plant also grows in Europe south-western Asia, and North America [20].

\section{Description}

5.1. Macroscopic. Fruit subspherical, purplish-black showing a "bloom" $(0.5-1.0 \mathrm{~cm}$ in diameter): at the base are six, small, pointed, bracts arranged in 2 whorls, occasionally 3 or 4 whorls present; apex shows triradiate mark and depression indicating the suture; three hard, triangular seeds are embedded in the fleshy mesocarp, having terebinthne odour and bitter taste.

5.2. Microscopic. Seed coat shows 2-3 layers of thin-walled cells which are externally covered by a thin cuticle and which are internally followed by thick-walled polygonal sclerenchymatous cells. Endosperm and embryo are not distinct. Outer layer of fruit shows 3-4 large cubic or tabular cells having thick, brown porous walls. Sarcocarp consists of large, thin-walled, elliptical, loosely coherent cells, containing prismatic crystals of calcium oxalate and drops of essential oil [21].

\section{Traditional Uses}

See Table 1.

\section{Phytochemical Screening}

Dried powder of J. communis stems (200 g) was successively extracted with petroleum ether chloroform and ethanol (soxhlet). The marc was obtained which was successively air dried. Water extract was successively obtained by boiling with distilled water $(2 \mathrm{~h})$. Than it was filtered, concentrated, and dried in an oven. After that all the extracts were dissolved in their relevant solvents and were screened for phytoconstituents [22] (Table 2).

\section{Chemical Constituents}

It contains various chemical constituents including flavonoids, volatile oil, and coumarins.

\subsection{Flavonoids}

8.1.1. Berries. They contain apigenin, rutin, luteolin, quercetin-3-O-arabinosyl-glucoside, quercetin-3-o-rhamnoside quercitrin, scutellarein, nepetin, amentoflavone, and bilobetin [3, 23-27] (Figure 2). 
TABLE 2: Phytochemical screening of J. communis [12].

\begin{tabular}{lrcccc}
\hline Serial number & Phytoconstituents & Petroleum ether extracts & Chloroform extracts & Methanol extracts & Aqueous extracts \\
\hline 1 & Alkaloids & - & + & + & + \\
2 & Flavonoids & - & + & + & + \\
3 & Glycosides & - & - & + & + \\
4 & Tannins and phenolic compounds & - & $+/+$ & + & + \\
5 & Steroids/triterpenoides & - & - & - & + \\
6 & Carbohydrates & - & - & - & - \\
7 & Proteins and amino acids & - & & - \\
\hline
\end{tabular}

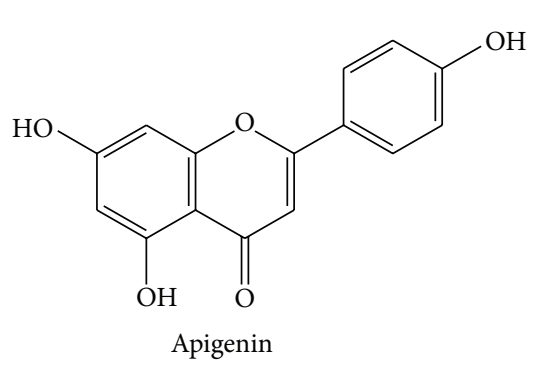

(a)<smiles></smiles>

(b)

Figure 2

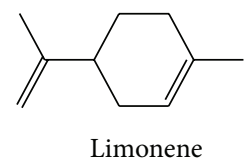

(a)

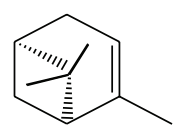

$\alpha$-Pinene

(b)<smiles>C=CC(=C)CCC=C(C)C</smiles>

(c)<smiles>O=C1O[C@H]([C@@H](O)CO)C(O)=C1O</smiles>

Ascorbic acid

(d)<smiles>O=COC(=O)c1ccccc1</smiles>

(e)<smiles>O=C(O)CC(O)C(=O)O</smiles>

Malic acid

(f)

Figure 3

8.1.2. Leaves. They contain the cupressuflavone, hinokiflavone, biflavones, isocryptomerin amentoflavone, and sciadopitysin. The seeds contain haemagglutinin. Plant also contains several labdane diterpenes and diterpenoids (methanolic extract) [28].

8.2. Volatile Oil. The juniper berry oil is largely comprised of monoterpene hydrocarbons such as $\beta$-pinene $(5.0 \%)$, $\alpha$-pinene $(51.4 \%)$, sabinene $(5.8 \%)$, myrcene $(8.3 \%)$, and limonene (5.1\%) [15] (Figure 3). The seeds and fruits of the plant contain $\mathrm{d}$ - $\alpha$-pinene, camphene, pectins, glycolic acid, malic acid, formic acid, acetic acid, cyclohexitol, terpene, proteins, fermentable sugars, wax, gum, ascorbic acid, dihydrojunene, $\beta$-pinene, hydrocarbon-junene, cadinene, juniper, and camphor [29].<smiles>O=c1ccc2ccc(O)cc2o1</smiles>

Figure 4

8.3. Coumarins. They contain umbelliferone; see Figure 4 [23].

8.4. Bicyclic Diterpenes. They contain imbricatolic acid, Junicedral, trans-Communic acid, diterpenes, isocupressic acid, aryltetralin, and lignan deoxypodophyllotoxin [6, 29]. Three 
new diterpene acids have been identified as 15-dien-18-oic acid, 7-oxo-13-epi-pimara-8, $7 \alpha$-hydroxysandaracopimaric acid [30-32].

\section{Pharmacological Activities}

9.1. Hepatoprotective Activity. The hepatoprotective activity of $J$. communis in rats was determined by given $\mathrm{CCl}_{4}$ administration for 9 days. In $\mathrm{CCl}_{4}$ treatment group was showed significant increase in serum glutamic oxaloacetic transaminase (SGOT), serum glutamic pyruvic transaminase (SGPT), total bilirubin (TB), and alkaline phosphatase (ALP) values when compared to control group. There was significant decrease in the level of SGPT, SGOT, TB, and ALP in silymarin treated group. The abnormal high level of SGOT, SGPT, ALP, and bilirubin observed was due to $\mathrm{CCl}_{4}$ induced hepatotoxicity. J. communis reduced the increased levels of serum SGPT, SGOT, ALP, and bilirubin, which showed protection against hepatic cells (ethanol and aqueous extract show better protection) [12].

9.2. Anti-Inflammatory Activity. Anti-inflammatory activity of $J$. communis fruit has determined using isolated cells and enzymatic test. The plant showed varying degree of activity at $0.2 \mathrm{mg} / \mathrm{mL}$ in prostaglandin test and $0.25 \mathrm{mg} / \mathrm{mL}$ in platelet activating factor (PAF) test (aqueous extract). $J$. communis showed 55\% prostaglandin inhibition and 78\% PAF-exocytosis inhibition. The PAF activity was measured by inducing exocytosis of elastase. All plant extracts were studied on thin layer chromatography eluted with ethyl acetate/methanol/water [9].

9.3. Antioxidant Activity. Antioxidant activity has reported the in vitro antioxidant activity of plant using different assays like DPPH scavenging, superoxide scavenging, ABTS radical cation scavenging, and hydroxyl radical scavenging. The antioxidant effects of the oil were confirmed by in vivo study and created the possibility of blocking the oxidation processes in yeast cells by increasing the activity of the antioxidant enzymes [15].

9.4. Antidiabetic and Antihyperlipidemic Activity. J. communis was reported to have antidiabetic and antihyperlipidemias activity in streptozotocin- (STZ-) nicotinamide induced diabetic rats. J. communis (methanolic extract, $100 \mathrm{mg} / \mathrm{kg}$ and $200 \mathrm{mg} / \mathrm{kg}$ p.o.) was administered except to the group that received (glibenclamide $10 \mathrm{mg} / \mathrm{kg}$ ). Biochemical estimation and fasting blood glucose levels were estimated on the 21st day. The methanolic extract of $J$. communis mediated significant $(P<0.01)$ reduction in blood glucose levels and increase in HDL levels in diabetic rats. Glibenclamide (standard drug) showed a significant decrease in the level of SGPT and SGOT. Methanolic extract of J. communis showed a significant anti diabetic and antihyperlipidemic activity [13].

9.5. Analgesic Activity. Banerjee and collaborators [11] reported the analgesic activity of $J$. communis using methanolic extract. The methanolic extract was given at a dose of $100 \mathrm{mg} / \mathrm{kg}$ and $200 \mathrm{mg} / \mathrm{kg}$ and evaluated for its analgesic activity. Acetylsalicylic acid was used as standard (100 mg/ $\mathrm{kg}$ ). In vivo the extract was evaluated by different tests like formalin test, acetic acid induced writhing, and tail flick tests. J. communis showed a significant $(P<0.01)$ and dose dependent effect on inhibition of writhing response and dose dependent inhibition in the late phase as compared to aspirin $(P<0.01)$, formalin test. The blocking effect of naloxone $(2 \mathrm{mg} / \mathrm{kg}$ i.p. $)$ confirms the central analgesic activity. The plant showed significant antinociceptive activity and it has been established that the methanolic extract of $J$. communis acts both peripherally and centrally [11].

9.6. Antibacterial Activity. The leaf extracts (methanol, ethanol, chloroform, and hexane aqueous) of J. communis were evaluatedagainst five pathogenic multidrug resistant bacteria (Erwinia chrysanthemi, Escherichia coli, Bacillus subtilis, Agrobacterium tumefaciens, and Xanthomonas phaseoli), by using disc diffusionmethod. It has been estimated that all extracts of leaves of $J$. communis were effective against the pathogenic bacteria except aqueous extract. The hexane extract showed more activity as compared to other extracts (hexane $>$ ethanol $>$ methanol $>$ chloroform extract). The methanolic extract of J. communis was found to be very effective as compared to standard antibiotics (ampicillin $10 \mathrm{mcg}$ and erythromycin $15 \mathrm{mcg}$ ) [17].

9.7. Antimicrobial Activity. The berries of J. communis were reported to have antimicrobial activity and volatile oils were analyzed by GC-FID and GC-MS. Its oil was investigated for its antimicrobial activity and the activity was tested against Escherichia coli, Staphylococcus aureus, Hafnia alvei, and Pseudomonas aeruginosa. DMF solution with three different concentrations of essential oil $(1,3$, and $5 \mathrm{mg} / \mathrm{mL}$ ) was prepared which were applied on disc for the measurement of the diameter of the zone of inhibition around the disc. The chromatographic analysis of the essential oil of $J$. communis allowed identifying 41 components which represent $96 \%$ of the oil total composition (Table 3 ). The main chemical constituents in J. communis were $\alpha$-cadinol (1.6\%), $\alpha$-pinene (36.2\%), $\beta$-myrcene (21.1\%), $\alpha$-humulene $(1.5 \%)$, epi- $\alpha$-bisabolol $(1.3 \%)$, germacrene D (2.2\%), spathulenol (1.4\%), and germacrene B (1.1\%). The present study shows the chemical composition of J. communis from east part of Kosova. J. communis was active against Escherichia coli, Staphylococcus aureus, and Hafnia alvei except Pseudomonas aeruginosa which is resistant to J. communis [14].

9.8. Antifungal Activity. The aerial parts of J. communis were isolated by hydrodistillation for their essential oil with 0.1 and $0.3 \%$ yield. The oils were then tested for their antifungal (in vitro) activity against two fungi, Rhizoctonia solani and Rhizopus stolonifer. The essential oils obtained from J. communis showed antifungal activity against both fungi: J. communis (EC50: 0.554 and $0.704 \mathrm{mg} / \mathrm{mL}$ ). The antifungal activity of $J$. communis is mainly due to the presence of high content of oxygenated monoterpenes [8]. 
TABLE 3: Essential oil components of J. communis L. [8].

\begin{tabular}{lcc}
\hline & $\mathrm{Rt}$ & $\%$ \\
\hline Monoterpene hydrocarbons & & \\
(i) $\alpha$-Pinene & 4.40 & 1.95 \\
(ii) dl-Limonene & 6.33 & 0.96 \\
(iii) $\alpha$-Pinene & 10.78 & 0.80 \\
(iv) $(+$ )-4-Carene & 12.44 & 3.86 \\
(v) Bicyclo[4.1.0]hept-2-ene,3,7,7-trimethyl & 12.81 & 0.71 \\
\hline & $\mathrm{Rt}$ & $\%$ \\
\hline Sesquiterpene hydrocarbons & & \\
(i) $\alpha$-Cedrene & 12.98 & 0.15 \\
(ii) $\alpha$-Cadina-4,9-diene & 13.08 & 0.93 \\
(iii) Cedrene & 13.64 & 4.04 \\
(iv) Gamma. 1-cadinene & 14.09 & 1.00 \\
\hline & $\mathrm{Rt}$ & $\%$ \\
\hline Oxygenated monoterpenes & & \\
(i) 1-Indanone & 7.60 & 1.15 \\
(ii) Linalool & 7.85 & 2.34 \\
(iii) $2,3,3$-Trimethyl-3-cyclopentene acetaldehyde & 8.35 & 2.09 \\
(iv) 5-Decene-1-ol & 10.89 & 2.60 \\
\hline & $\mathrm{Rt}$ & $\%$ \\
\hline Oxygenated sesquiterpenes & 18.94 & 2.79 \\
(i) Cedrene epoxide & & \\
\hline
\end{tabular}

9.9. Antimalarial Activity. The leaves and twigs (stems) of eight plants were isolated for their essential oil by hydrodistillation method (Juniperus communis, Artemisia vulgaris, Myrtus communis, Lavandula angusti/olia, Eucalyptus globulus, Rosmarinus officinalis, Origanum vulgare, and Salvia officinalis) and were analyzed by GC-FID and GC-MS. The essential oil obtained from these plants was then tested for their antimalarial activity on Plasmodium falciparum. There were two strains of Plasmodium falciparum: FcBl Columbia and a Nigerian chloroquine-sensitive strain. Two concentrations ranged from $150 \mu \mathrm{g} / \mathrm{mL}$ to $1 \mathrm{mg} / \mathrm{mL}$ showed $50 \%$ inhibition of the growth of the parasite (in vitro) and the effect was obtained after 24 and $72 \mathrm{~h}$. Myrtus communis and Rosmarinus officinalis oils at a concentration ranged from 150 to $270 \mu \mathrm{g} / \mathrm{mL}$ showed best result against Plasmodium falciparum [33].

9.10. Antihypercholesterolemic Activity. J. communis fruit oil has been evaluated for its antihypercholesterolemic activity. The biochemical parameters and the histopathologic effects on kidney tissue were evaluated. Healthy Wistar albino rats of 200-250 gm in weight were used for this study. The rats were divided into 5 groups; first group is control group in which the animal was fed with normal pellet chow. The second group is cholesterol group which was fed with pellet chow containing $2 \%$ of cholesterol, and the third group is J. communis (JCL) group which was further divided into three subgroups 50 JCL, 100 JCL, and 200 JCL groups which were fed with 50, 100, and $200 \mathrm{mg} / \mathrm{kg} J$. communis oil, with addition to the $2 \%$ cholesterol-containing pellet chow. JCL was administered by a gavage needle (dissolved in $0.5 \%$ sodium carboxy methyl cellulose (SCMC). After 30 days blood and kidney tissue samples were taken and biochemical estimation and histopathological investigation were done. The $200 \mathrm{mg} / \mathrm{kg}$ JCL group showed a significant increase in blood urea nitrogen (BUN) and creatinine levels. The cholesterol group showed a significant increase in Ox-LDL levels. When the cholesterol was given along with $200 \mathrm{mg} / \mathrm{kg}$ J. communis then there was no significant increase in the level of Ox-LDL. So the study showed its antihypercholesterolemic effect [16].

9.11. Anticataleptic Activity. Anticataleptic study was carried out to evaluate the effects of methanolic extract of J. communis (MEJC) leaf in reserpine induced catalepsy in rats. Catalepsy was induced by intraperitoneal (i.p.) administration of reserpine $(2.5 \mathrm{mg} / \mathrm{kg}$, i.p.). The methanolic extract at 100 and $200 \mathrm{mg} / \mathrm{kg}$ (i.p.) was screened for its efficacy against reserpine induced catalepsy in rats. The MEJC extract was found to reduce catalepsy significantly $(P<0.001)$ as compared to the reserpine treated rats; maximum reduction was observed at a dose of $200 \mathrm{mg} / \mathrm{kg}$ [18].

9.12. Neuroprotective Activity. Neuroprotective activity of $J$. communis (MEJC) was evaluated in chlorpromazine (CPZ) induced Parkinson's model in rats. The two doses (100 and $200 \mathrm{mg} / \mathrm{kg}$, i.p.) have been selected on the basis of lethal dose $\left(L_{50}\right)$ in mice. The plant was evaluated for various behavior parameters like catalepsy (bar test), muscle rigidity (rot rod test), and locomotor activity (actophotometer) and its effect on biochemical parameters (TBARS, GSH, nitrite, and total protein) in rats brain. J. communis showed a significant $(P<$ 0.001 ) neuroprotective effect of MEJC against CPZ induced Parkinson's like symptoms or anti-Parkinson's activity [2].

\section{Conclusion}

The extensive literature survey revealed that $J$. communis L. is an important medicinal plant due to its traditional uses to treat diseases and presence of many active chemical constituents which are responsible for various medicinal and pharmacological properties. Further evaluation needs to be carried out on J. communis L. in order to confirm its medicinal uses and development of formulations containing this plant for their practical clinical applications, which can be used for the welfare of mankind.

\section{Conflict of Interests}

The authors declare that there is no conflict of interests regarding the publication of this paper.

\section{References}

[1] S. Kakkar and S. Bais, "A review on protocatechuic acid and its pharmacological potential," ISRN Pharmacology, vol. 2014, Article ID 952943, 9 pages, 2014.

[2] N. Rana and S. Bais, Neuroprotective effect of J. communis in Parkinson disease induced animal models [M.S. thesis in 
Pharmacy], Pharmacology Department, Punjab Technical University, Punjab, India, 2014.

[3] E. Lamer Zarawska, "Biflavonoids in Juniperus species (Cupressaceae)," Polish Journal of Pharmacology and Pharmacy, vol. 27, no. 1, pp. 81-87, 1975.

[4] V. Tandon, B. Kapoor, and B. M. Gupta, "Herbal drug research in India: a trend analysis using IJP as a marker (1995-August 2003)," Indian Journal of Pharmacology, vol. 36, no. 2, pp. 99100, 2004.

[5] Steven D. Ehrlich; NMD, Solutions Acupuncture, a Private Practice Specializing in Complementary and Alternative Medicine, Steven D. Ehrlich, NMD, Healthcare Network, Phoenix, Ariz, USA, 2009.

[6] A. M. L. Seca and A. M. S. Silva, "The chemical composition of the Juniperus Genus (1970-2004)," in Recent Progress in Medicinal Plants, vol. 16 of Phytomedicines, pp. 402-522, 2005.

[7] N. Gumral, D. D. Kumbul, F. Aylak, M. Saygin, and E. Savik, "Juniperus communis Linn oil decreases oxidative stress and increases antioxidant enzymes in the heart of rats administered a diet rich in cholesterol," Toxicology and Industrial Health, 2013.

[8] D. Modnicki and J. Łabędzka, "Estimation of the total phenolic compounds in juniper sprouts (Juniperus communis, Cupressaceae) from different places at the kujawsko-pomorskie province," Herba Polonica, vol. 55, no. 3, 2009.

[9] H. Tunon, C. Olavsdotter, and L. Bohlin, "Evaluation of antiinflammatory activity of some Swedish medicinal plants. Inhibition of prostaglandin biosynthesis and PAF-induced exocytosis," Journal of Ethnopharmacology, vol. 48, no. 2, pp. 61-76, 1995.

[10] M. A. Abbassy and G. I. Marei, "Antifungal and chemical composition of essential oils of J. communis and Thymus vulgaris against two phytopathogenic fungi," Journal of Applied Sciences Research, vol. 9, no. 8, pp. 4584-4588, 2013.

[11] S. Banerjee, A. Mukherjee, and T. K. Chatterjee, "Evaluation of analgesic activities of methanolic extract of medicinal plant Juniperus communis Linn," International Journal of Pharmacy and Pharmaceutical Sciences, vol. 4, no. 5, pp. 547-550, 2012.

[12] Manvi and G. P. Garg, "Screening and evaluation of pharmacognostic, phytochemical and hepatoprotective activity of $J$. communis L. Stems," International Journal of Pharma and Bio Sciences, vol. 1, no. 3, 2010.

[13] S. Banerjee, H. Singh, and T. K. Chatterjee, "Evaluation of anti-diabetic and anti-hyperlipidemic potential of methanolic extract of Juniperus Communis (L.) in streptozotocinnicotinamide induced diabetic rats," International Journal of Pharma and Bio Sciences, vol. 4, no. 3, pp. P10-P17, 2013.

[14] S. Pepeljnjak, I. Kosalec, Z. Kalodera, and N. Blažević, "Antimicrobial activity of juniper berry essential oil (Juniperus communis L., Cupressaceae)," Acta Pharmaceutica, vol. 55, no. 4, pp. 417-422, 2005.

[15] M. Hoferl, I. Stoilova, E. Schmidt et al., "Chemical composition and antioxidant properties of Juniper Berry (J. communis L.) Essential oil. Action of the essential oil on the antioxidant protection of Saccharomyces cerevisiae model organism," Antioxidants, vol. 3, no. 1, pp. 81-98, 2014.

[16] M. Akdogan, A. Koyu, M. Ciris, and K. Yildiz, "Anti-hypercholesterolemic activity of J. communis Oil in rats: a biochemical and histopathological investigation," Biomedical Research, vol. 23, no. 3, pp. 321-328, 2012.

[17] S. C. Sati and S. Joshi, "Antibacterial potential of leaf extracts of Juniperus communis L. from Kumaun Himalaya," African
Journal of Microbiology Research, vol. 4, no. 12, pp. 1291-1294, 2010.

[18] S. Bais, S. Gill, and N. Rana, "Effect of J. communis extract on reserpine induced catalepsy," Inventi Rapid: Ethnopharmacology, vol. 2014, no. 4, pp. 1-4, 2014.

[19] S. Vichi, M. Riu-Aumatell, M. Mora-Pons, J. M. Guadayol, S. Buxaderas, and E. López-Tamames, "HS-SPME coupled to GC/MS for quality control of Juniperus communis L. berries used for gin aromatization," Food Chemistry, vol. 105, no. 4, pp. 1748-1754, 2007.

[20] P. K. Sharma and B. Lal, "Ethnobotanical notes on some medicinal and aromatic plants of Himachal Pradesh," Indian journal of Traditional Knowledge, vol. 4, no. 4, pp. 424-428, 2005.

[21] A. K. Nandkarni, Indian Materia Medica, Popular Prakashan Private Limited, Bombay, India, 1976.

[22] N. R. Farnsworth, "Biological and phytochemical screening of plants," Journal of Pharmaceutical Sciences, vol. 55, no. 3, pp. 225-276, 1966.

[23] E. Lamer-Zarawska, "Phytochemical studies on flavonoids and other compounds of juniper fruits," Polish Journal of Chemistry, vol. 54, no. 2, pp. 213-219, 1980.

[24] A. Hiermann, A. Kompek, J. Reiner, H. Auer, and M. SchubertZsilavecz, "Investigation of flavonoid pattern in fruits of juniperus communis L," Scientia Pharmaceutica, vol. 64, no. 3-4, pp. 437-444, 1996.

[25] M. Kowalska, "Chemical composition of common juniper (J. communis L.) fruits," Roczniki Akademii Rolniczej w Poznaniu, vol. 117, pp. 61-64, 1980.

[26] E. Lamer-Zarawska, "Flavonoids of J. communis L”, Roczniki Chemii, vol. 51, no. 11, pp. 2131-2137, 1977.

[27] M. Ilyas and N. Ilyas, "Biflavones from the leaves of J. communis and a survey on biflavones of the Juniperus genus," Ghana Journal of Chemistry, vol. 1, no. 2, pp. 143-147, 1990, (CA 252113p, 1991, vol. 115).

[28] C. P. Khare, Indian Medicinal Plants, Springer Science, New York, NY, USA, 2007.

[29] K. Chandra, B. G. Chaudhari, B. P. Dhar et al., Database in Medicinal Plants Used in Ayurveda, vol. 5, 3rd edition, 2007.

[30] J. de Pascual Teresa, A. F. Barrero, L. Muriel, A. San Feliciano, and M. Grande, "New natural diterpene acids from Juniperus communis," Phytochemistry, vol. 19, no. 6, pp. 1153-1156, 1980.

[31] P. S. Chatzopoulou and S. T. Katsiotis, "Chemical investigation of the leaf oil of Juniperus communis L," Journal of Essential Oil Research, vol. 5, no. 6, pp. 603-607, 1993.

[32] A. Y. Gordien, A. I. Gray, S. G. Franzblau, and V. Seidel, "Antimycobacterial terpenoids from Juniperus communis L. (Cuppressaceae)," Journal of Ethnopharmacology, vol. 126, no. 3, pp. 500-505, 2009.

[33] G. Milhau, A. Valentin, F. Benoit et al., "In vitro antimalarial activity of eight essential oils," Journal of Essential Oil Research, vol. 9, no. 3, pp. 329-333, 1997. 

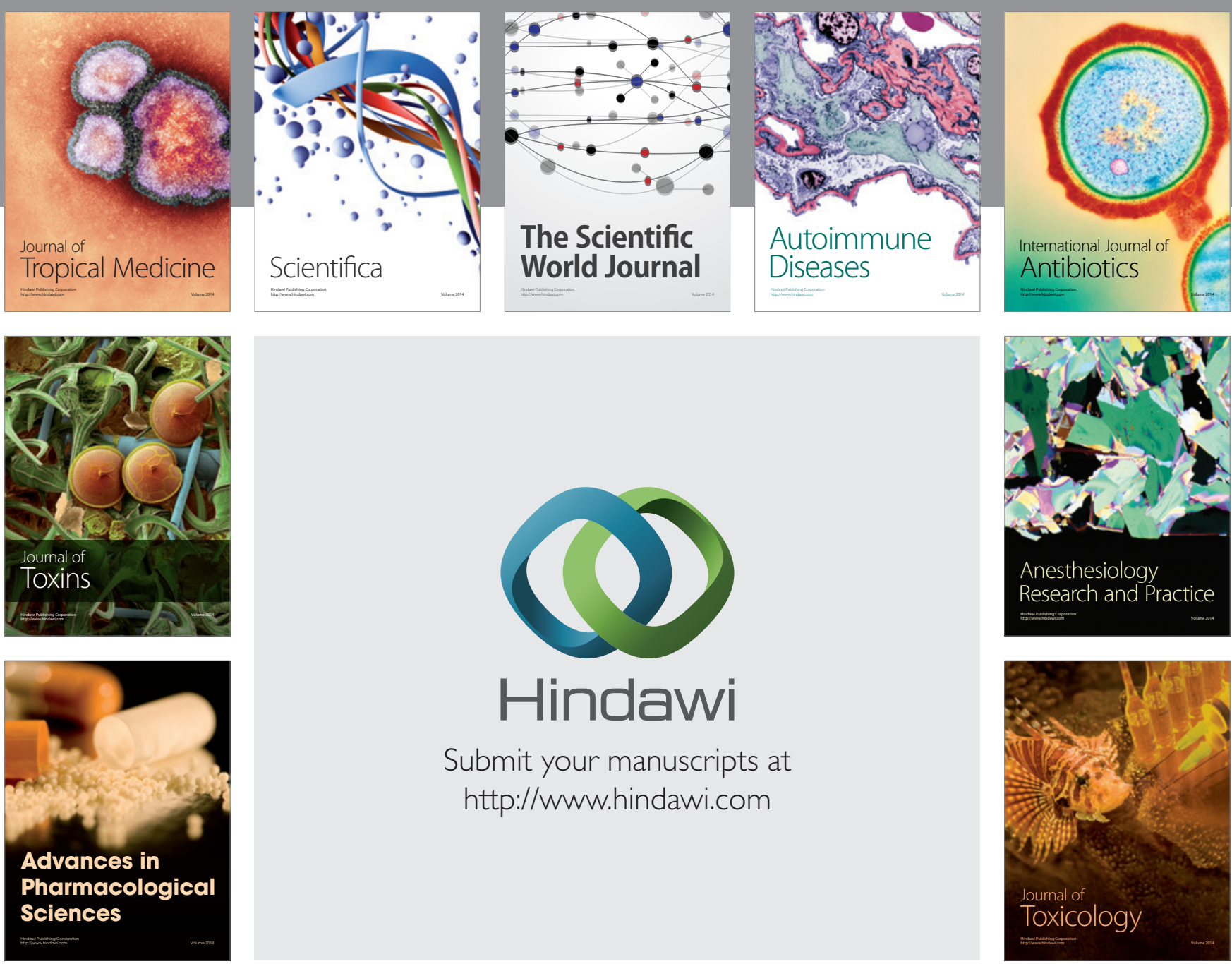

\section{Hindawi}

Submit your manuscripts at

http://www.hindawi.com
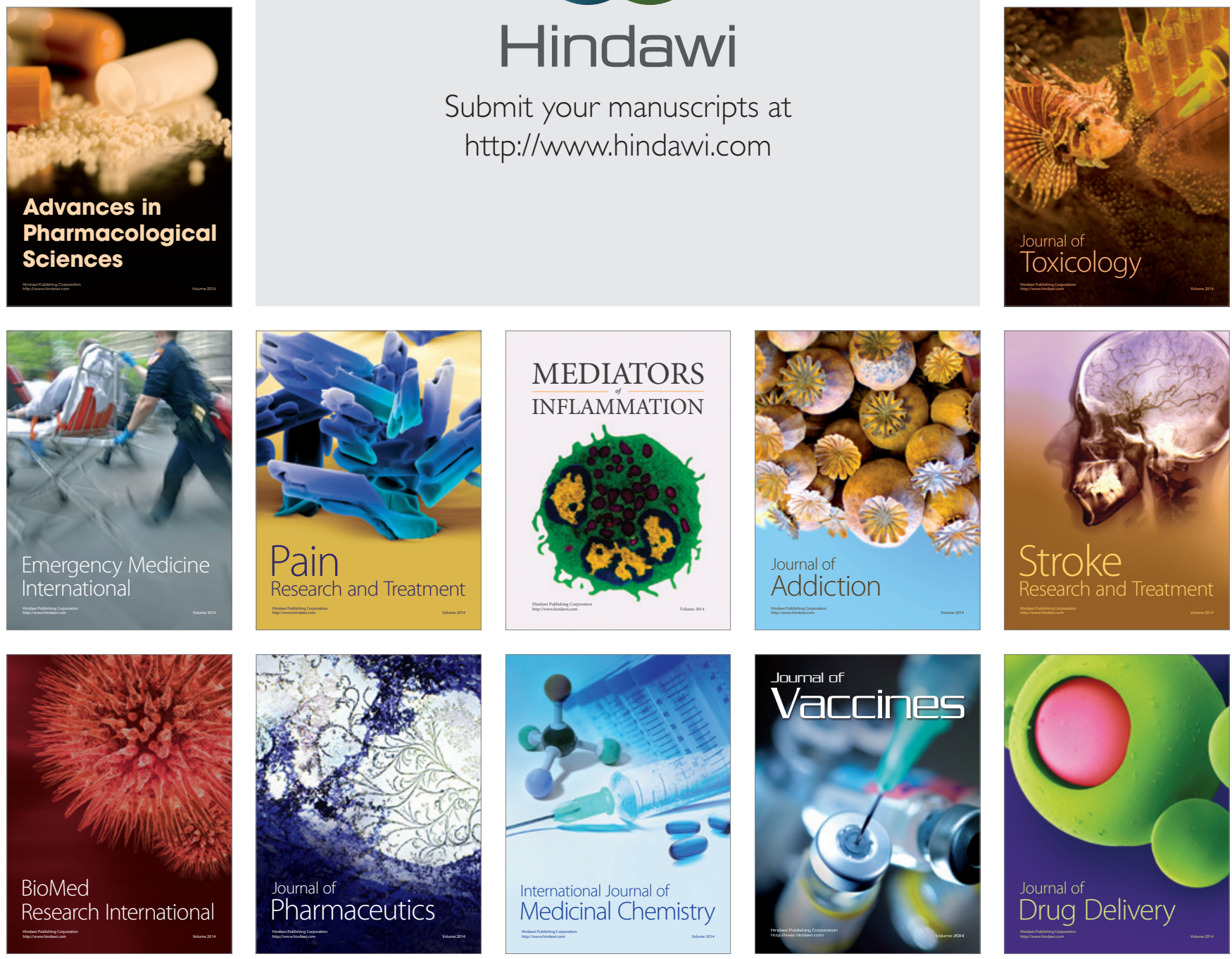